\title{
Effects of Large and Small Transections of the Preoptic- Hypothalamic Region on Hydromineral Regulation in Rats
}

\author{
Hiroshi KAWABE, Oswaldo U. LOPES*, \\ Cheryl L. CHERNICKY**, Karen B. BROSNIHAN**, \\ TAKaO SARUTA ANd Carlos M. FERRARIO**
}

Department of Internal Medicine, School of Medicine, Keio University, Tokyo 160, Japan, *Department of Physiology and Biophysics, Instituto de Ciencias Biomedicas, Universidade de San Paulo, Brazil, **Section of Cardiovascular Neurobiology, Department of Brain and Vascular Research, The Cleveland

Clinic Foundation, Cleveland, $\mathrm{OH} 44106$, U.S.A.

\begin{abstract}
To further elucidate the role of the preoptic-hypothalamic region in fluid and electrolyte balance we studied the effect of surgical preoptic-hypothalamic disconnection using either a large (preoptic-hypothalamic disconnection) or a small (medial preoptic-hypothalamic disconnection) microknife. Both the large and small cuts seemed to transect the posterior projection originating in the periventricular tissue surrounding the anteroventral third ventricle (AV3V) and extending to supraoptic nucleus, but the supraoptic-neurohypophysial pathway was severed only by the large cut. Seven-day metabolic studies showed a disruption in hydromineral balance only in large cut rats; they had increased water intake and urine volume on day 1, a near-recovery of function on days 2 and 3 , and polydipsia and polyuria on days 4 to 7 . There was no difference between small cut rats and sham-operated rats in metabolic measurements. The large cut rats also had sustained hypernatremia and hyperosmolality, which was enhanced after water restriction for $48 \mathrm{~h}$ but was not accompanied by an increase in plasma arginine vasopressin. Our data therefore suggest that the efferent fibers running caudally from the $\mathrm{AV} 3 \mathrm{~V}$ are not involved in mediation of the hydromineral regulation of the AV3V.
\end{abstract}

Key words: Preoptic-hypothalamic disconnection, AV3V-SON pathway, Supraoptic-neurohypophysial pathway, Sodium excretion, Arginine vasopressin.

(Endocrine Journal 40: 249-256, 1993)

RECENTLY a number of investigations have evaluated the role of the endocrine hypothalamus and its associated afferent and efferent pathways on the hemodynamic, humoral, and hormonal events. Structures within the tissue surrounding the anteroventral portion of the third ventricle (AV3V) of the rat are of particular importance since destruction of this area has been shown to cause a disruption in hydromineral balance and block the development of various forms of experimental hypertension [1-4]. Bealer et al. [5, 6]

Received: August 25, 1992

Accepted: February 18, 1993

Correspondence to: Dr. Hiroshi KAWABE, Department of Internal Medicine, School of Medicine, Keio University, 35

Shinanomachi, Shinjuku-ku, Tokyo 160, Japan have reported that rats with coronal knife cuts between the level of the organum vasculosum of the lamina terminalis (OVLT) and the level of the supraoptic nucleus (SON) exhibited an increase in water intake and urine volume. They suggested that a neural pathway coursing through this brain region is critical for fluid regulation. Castro $e t$ al. [7] also have previously reported in rats that a preoptic-hypothalamic microknife cut produces a triphasic pattern of water intake. Initial polydipsia gave way to brief normodipsia, followed by permanent polydipsia. However, the metabolic changes associated with altered water intake have not yet been studied. Thus the objective of this study was to characterize, by means of a microknife cut, the effects of partial denervation of the preoptic 
region of the hypothalamus on the regulation of hydromineral balance and arginine vasopressin (AVP) secretion in the rat. The following questions were addressed: first, do these knife cuts produce alterations in metabolic indices other than polydipsia? Second, how do these lesioned rats respond to water restriction?

\section{Materials and Methods}

\section{Animals}

Sixty-five male Sprague-Dawley rats (Hilltop Lab Animals) weighing between 210 to $300 \mathrm{~g}$ at the time of surgery were used in these experiments. The rats were housed individually in Nalgene (Nalge) metabolic cages in a laboratory maintained at a constant temperature $\left(22^{\circ} \mathrm{C}\right)$ with a $12 \mathrm{~h}$ light $12 \mathrm{~h}$ dark cycle. The rats were allowed free access to water and food (Rodent Lab Chow 5001). All procedures were conducted according to the Recommendations from the Declaration of Helsinki and the Guiding Principles in the Care and Use of Animals.

\section{Animal Surgery}

Microknife cuts: After sodium pentobarbital anesthesia $(50 \mathrm{mg} / \mathrm{kg}$ intraperitoneally, Nembutal, Abbott Laboratories) the rats were positioned in a stereotaxic head holder (David Kopf), and the incisor bar of the instrument was positioned $5 \mathrm{~mm}$ below the level of the interaural line. This procedure ensured that the intracranial surface of the sphenoid was in a horizontal plane [8]. Following exposure of the brain surface the sagittal venous sinus was sectioned between ligatures (6-0 Prolene).

Two different types of preoptic-hypothalamic disconnection were performed. In one of these $(n=29)$ the microknife was inserted into the brain according to the method of Halasz and Pupp [8]. The cutting edge of the microknife measured 3 $\mathrm{mm}$ in height and had a radius of $1.5 \mathrm{~mm}$. The axis of the microknife was positioned $3 \mathrm{~mm}$ caudal to the bregma and its tip was positioned $1.5 \mathrm{~mm}$ caudal to the bregma. The microknife was then lowered into the brain along the midline until the tip touched the base of the skull. Once this point was reached, the microknife was rotated $90^{\circ}$ to the right and $90^{\circ}$ to the left. This type of lesion will hereafter be termed preoptic-hypothalamic disconnection (POD). The second type of lesion (medial preoptic-hypothalamic disconnection, mPOD, $n=13$ ) was performed with a microknife that had a radius of $0.75 \mathrm{~mm}$ (3 $\mathrm{mm}$ in height). For these experiments the tip of the microknife was positioned $1.5 \mathrm{~mm}$ caudal to the bregma and rotated $90^{\circ}$ each side. All sham-operated rats $(n=23)$ were animals in which the tip of the microknife was lowered $5 \mathrm{~mm}$ from the skull surface but not rotated. Postoperatively, all rats received a single dose of penicillin G $(100,000$ units intramuscularly, Butler).

Arterial cannulation: On the last day of the experiments both lesioned and sham-operated rats were lightly anesthetized with methoxyflurane (Penthrane, Abbott Laboratories) and a carotid artery was cannulated (with PE-60 polyethylene tubing) to record blood pressure and obtain blood samples. The animals were then allowed to recover from the anesthesia for a minimum of $5 \mathrm{~h}$ or until they exhibited no ataxia. After recovery each animal was placed in a plexiglass holder (Narco Bio-Systems) and acclimatized to the new environment for $1 \mathrm{~h}$.

\section{Experimental protocols}

Twenty-nine POD, $13 \mathrm{mPOD}$, and 23 shamoperated controls were placed in Nalgene metabolic cages for 2 days prior to receiving microknife cuts or sham operations, and for 4 days (8 POD and 5 sham-operated) or 7 days (21 POD, 13 mPOD, and 18 sham-operated) following surgery. Daily measurements of water intake, urine volume, and food intake were recorded. Water intake was measured on inverted $100 \mathrm{ml}$ graduated burettes with a metal spout projecting into the cage. Urine was collected in graduated cylinders placed beneath the metabolic cages. Daily urinary sodium and potassium excretion were measured in 16 POD and 14 sham-operated rats. Sodium and potassium intakes were calculated as the product of the ion concentration in the food (sodium $=128$ $\mu \mathrm{Eq} / \mathrm{g}$; potassium $=275 \mu \mathrm{Eq} / \mathrm{g}$ ), and the amount of food consumed.

After completion of 4 or 7 days of metabolic study, blood pressure measurements and blood samplings were performed. Blood pressure was measured in conscious rats that were semi- 
restrained in a plexiglass holder. The carotid artery cannula was filled with heparinized saline and connected to a pressure transducer; blood pressure was recorded on a direct writing polygraph (Gould Inc., Cleveland, $\mathrm{OH}$ ). Pulse pressure was displayed on one channel, mean arterial pressure (MAP) on a second channel and heart rate (HR) on a third channel. Heart rate was electronically recorded with a cardiotachometer (Bio-Tech, Gould) triggered by the arterial pressure pulse. Blood samples $(2.5 \mathrm{ml})$ were obtained through the catheter implanted in the carotid artery and were collected in two chilled heparinized test tubes. The same volume $(2.5 \mathrm{ml})$ of normal saline was replaced just after each blood sampling. After separation by centrifugation at $4^{\circ} \mathrm{C}$, one plasma $(0.5 \mathrm{ml})$ was analyzed for osmolality, total protein, sodium and potassium. Another plasma sample $(0.5 \mathrm{ml})$ was stored at $-20^{\circ} \mathrm{C}$ for extraction and measurement of AVP.

After completion of the 7-day metabolic study, 8 out of 21 POD and 6 out of 18 sham-operated control rats were deprived of water for $48 \mathrm{~h}$ and continued to have daily urine volume and food intake measured for another 2 days. In these 14 rats, daily body weight was also measured during the experimental period. After completion of $48 \mathrm{~h}$ water restriction, blood pressure measurements and blood samplings were performed as described before.

\section{Biochemical techniques}

Plasma and urine sodium and potassium levels were determined by flame photometry; plasma and urine osmolality were measured by freezing point depression (Advanced Digimatic Osmometer, Model 3DII, Advanced). The concentration of AVP in plasma was measured by the radioimmunoassay described elsewhere [9].

\section{Histological techniques}

At the end of each experiment the brain was carefully removed from the skull and immersed in $10 \%$ buffered formalin for 1 week. At the end of this period the brain was transferred to $10 \%$ formalin containing $30 \%$ sucrose for storage. Fifty $\mu \mathrm{m}$ thick (frozen) sections of brain tissue were cut in either the coronal or horizontal plane. All sections were mounted and stained with cresyl violet for localization and reconstruction of the microknife cuts.

\section{Statistical analyses}

All values are reported as the means \pm SEM. Analysis of variance was performed to assess differences among groups, followed by the Dunnett's test for individual comparisons. The Student's $t$ test was also performed when applicable. Linear regression analyses were determined by the least-squares method. Differences were considered statistically significant when $P<0.05$.

\section{Results}

Histological examination of semicircular lesions produced by the larger microknife cut (POD) (Fig. 1) showed that the preoptic region, the medial forebrain bundle and the supraoptic region were disconnected from caudal and medial structures, specifically the anterior hypothalamus, median eminence and neurohypophysis. In contrast, the small microknife cut (mPOD) (Fig. 1) transected pathways traversing from the AV3V to hypothalamic structures. However, mPOD lesion spared the medial forebrain bundle as well as a major portion of the fibers that connected the supraoptic region to the neurohypophysis. There was no damage to the paraventricular nuclei (PVN) in any of the animals examined.

Presurgery values for water intake, urine volume, urine osmolality, food intake, and urinary sodium and potassium excretion were nearly the same in sham and both lesioned rats (Figs. 2, 3). Twenty-four hours after operation, water intake increased markedly in the POD rats but decreased in the rats with mPOD and sham operation. On the second and third days, water intake in POD rats returned toward control values, but after this time it became progressively greater. These changes in water intake were accompanied by parallel changes in urine volume and a sustained decrease in urine osmolality. Sham-operated and both groups of lesioned rats exhibited similar patterns of food intake during the 7-day experimental period, decreasing on the day after surgery, then gradually returning toward normal. A similar pattern was seen in changes in body weight (data not shown). There was no difference 

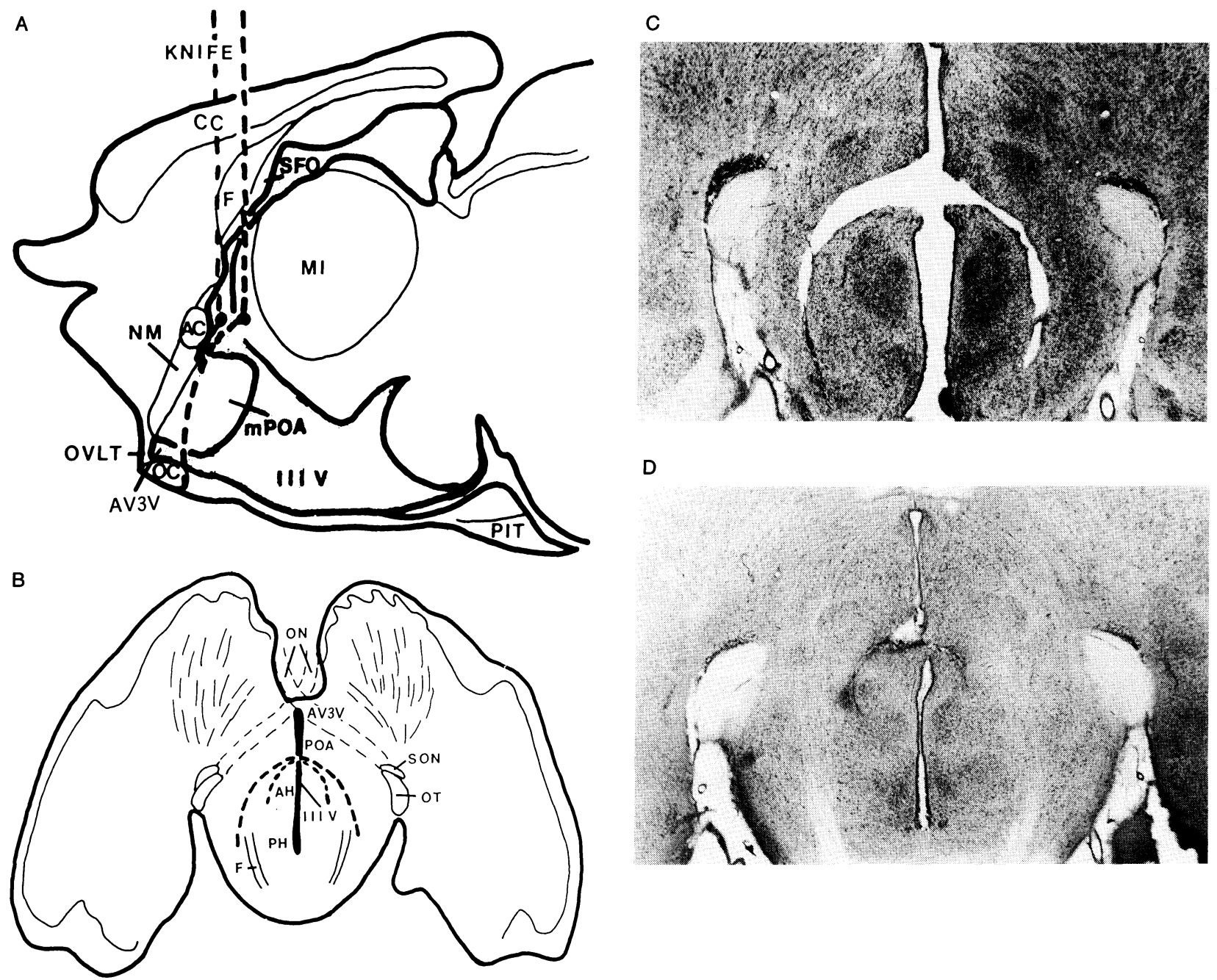

Fig. 1. The locations of the microknife cuts are illustrated schematically and with photomicrographs of cresyl violet-stained brain sections. A: diagram of a sagittal section of the rat brain. B: diagram of a horizontal section of the rat brain. C: horizontal section of preoptic-hypothalamic disconnection (radius: $1.5 \mathrm{~mm}$; height: $3 \mathrm{~mm}$ ). D: horizontal section of medial preoptic-hypothalamic disconnection (radius: $0.75 \mathrm{~mm}$; height: $3 \mathrm{~mm}$ ). The dotted arcs correspond to two types of disconnection (large microknife cut; preoptic-hypothalamic disconnection). Abbreviations: AC, anterior commissure; $\mathrm{AH}$, anterior hypothalamic nucleus; AV3V, anteroventral third ventricle; CC, corpus callosum; F, Fornix; MI, massa intermedia; mPOA, medial preoptic area; NM, nucleus medianus; OC, optic chiasm; ON, optic nerve; OT, optic tract; OVLT, organum vasculosum of the lamina terminalis; PH, posterior hypothalamic nucleus; PIT, pituitary; POA, preoptic area; SFO, subfornical organ; SON, supraoptic nucleus; III V, third cerebral ventricle.

between $\mathrm{mPOD}$ rats and sham-operated rats in water intake, urine volume or urine osmolality. In sham-operated rats, the fraction of dietary sodium excreted in the urine was significantly decreased only on the second and third days after surgery (Fig. 3). Preoptic-hypothalamic disconnection rats, on the other hand, exhibited a decreased urinary sodium excretion from the first day after surgery and it did not return to the control value until the sixth day after surgery. The fraction of dietary potassium excreted in the urine on the first day after surgery increased in both sham-operated and POD rats. From the second day, the amount of potassium excreted in the urine returned to control values in both groups. On the fourth day after operation hypernatremia and hyperosmolality were present in POD rats (Table 1). These changes were still present by the seventh day after the lesion. Furthermore, there were significantly positive correlations between the amount of water intake on the seventh day and either plasma sodium $(\mathrm{r}=0.74, P<0.01)$ or plasma osmolality 

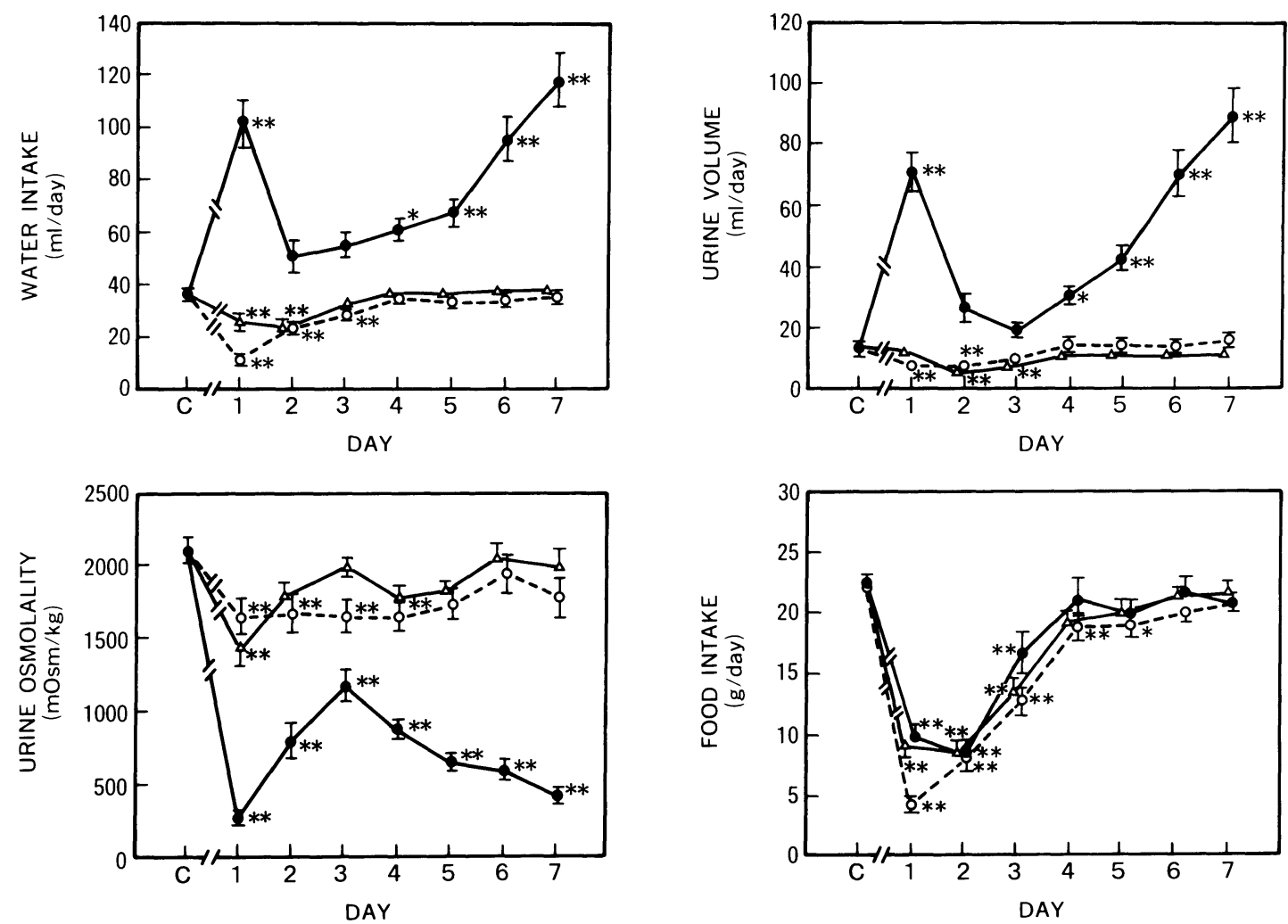

Fig. 2. Water intake, urine volume, urine osmolality, and food intake before and for 7 days after operation for the three groups of rats. Preoptic-hypothalamic disconnection (closed circle and solid line) caused significant increases in water intake and urine volume and a significant decrease in urine osmolality compared with medial preoptic-hypothalamic disconnection (open triangle and solid line) and sham-operated (open circle and dotted line) rats. Values are the means \pm SEM. ${ }^{*}, * *, P<0.05,0.01$ when experimental values are compared with the control values $(\mathrm{C})$.

$(\mathrm{r}=0.79, P<0.01)$ measured on the seventh day only in POD rats. These positive correlations were not seen in sham-operated rats, or in POD rats on the fourth day of the metabolic study. In spite of a sustained increase in plasma sodium and osmolality, the level of plasma AVP in POD rats did not increase (Table 1). The concentration of total protein in the plasma and hematocrit were not different when compared with the sham-operated rats on the fourth and seventh days (data not shown). Resting MAP and HR on the seventh day after surgery were not different among the 3 groups (data not shown).

In response to water restriction, sham-operated rats excreted concentrating urine, but POD rats failed to have this normal response (Table 2). It should be noted, however, that these rats are capable of producing relatively concentrated urine $(764 \pm 90 \mathrm{mOsm} / \mathrm{kg})$ after $48 \mathrm{~h}$ of water restriction. Although body weight in both groups was significantly decreased after water restriction, there was no significant difference in the fall in body weight between the groups (Table 2). A rise in plasma AVP after water restriction was seen in sham but not in the POD rats (Table 1). Plasma sodium and plasma osmolality were further increased in the POD rats (Table 1). Plasma total protein and hematocrit were increased after water restriction in both groups, but there was no difference between groups (data not shown). Although resting MAP and HR on the seventh day after surgery were not different among the 3 groups, POD rats had hypotension $(84 \pm 5 \mathrm{~mm} \mathrm{Hg}, \mathrm{n}=8)$ and bradycardia $(295 \pm 25$ beats/min, $\mathrm{n}=8)$ after $48 \mathrm{~h}$ of water restriction compared to sham-operated rats ( $142 \pm 3 \mathrm{~mm} \mathrm{Hg}, 464 \pm 7$ beats $/ \mathrm{min}, \mathrm{n}=6, P<0.01$ ).

\section{Discussion}

The periventricular tissue that surrounds the AV3V has been implicated in mediating AVP 

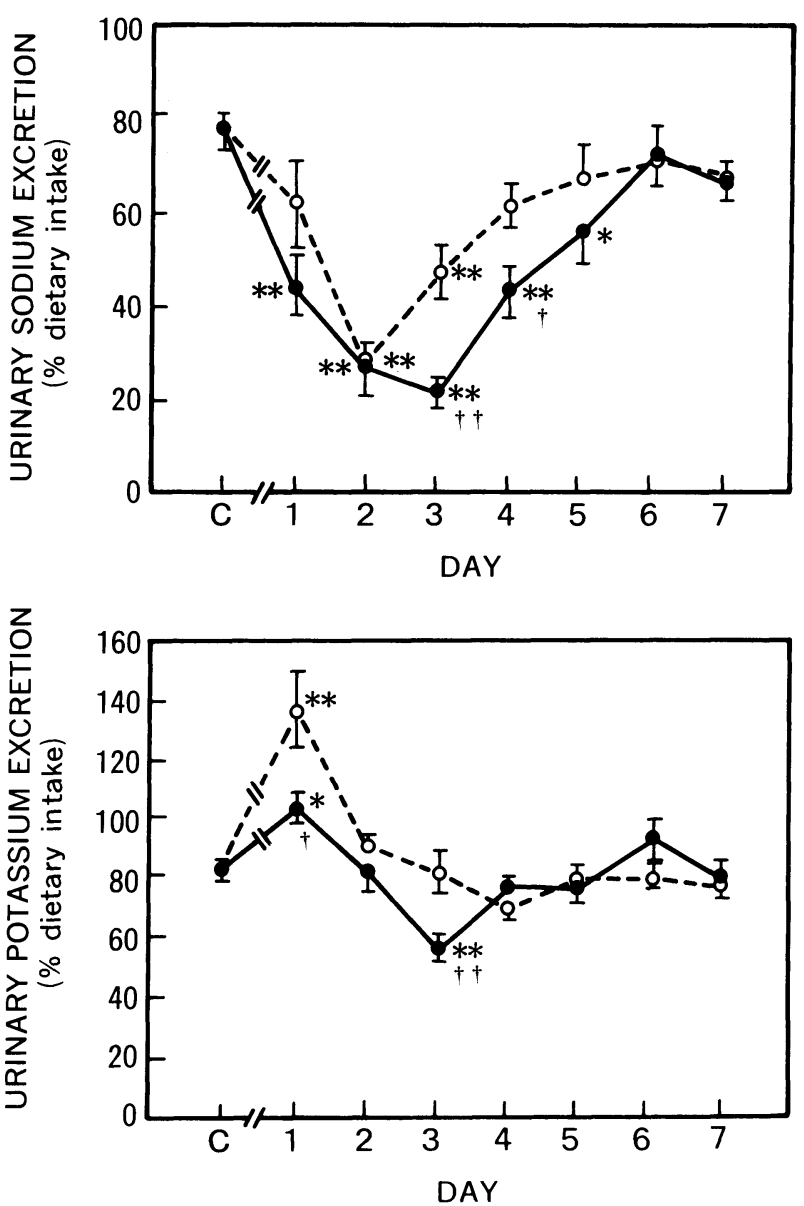

Fig. 3. The percent of dietary sodium and dietary potassium excreted in urine before and for 7 days after operation for the two groups of rats. Preoptic-hypothalamic disconnection (closed circle and solid line) showed an early decrease and a delayed recovery in sodium excretion compared with sham-operated rats (open circle and dotted line). Values are the means \pm SEM. *, **, $P<0.05,0.01$ when experimental values are compared with the control values $(\mathrm{C}) .^{\dagger},{ }^{\dagger \dagger}, P<0.05,0.01$ when experimental values are compared with shamoperated rats.

release in response to osmotic stimulation $[1,3,4]$. It has also been proposed that a neural pathway from AV3V to SON participates in both fluid balance and cardiovascular regulation $[3,4,10]$. In addition, it has been suggested that AV3V lesions interrupt neural inputs that stimulate the magnocellular system to release AVP [11]. From histological examinations, both POD and mPOD lesions spared the AV3V region, but the connection of the AV3V region with the hypothalamus, especially the fibers running caudally from the AV3V, seemed to be severed by either a large or small microknife. The POD lesion also transected the supraoptic-neurohypophysial pathway and the direct connections between the PVN and the neurohypophysis.

In this study, the mPOD lesion which spared the supraoptic-neurohypophysial pathway but severed the caudal efferents from the AV3V did not have any effect on hydromineral balance. Moreover, rats which received a unilateral (either side) POD lesion exhibited similar metabolic changes to those in sham-operated rats (unpublished observations). It is therefore possible to consider that the efferent fibers running caudally from the AV3V, a key area of hydromineral regulation, are not critically involved in mediation of the AV3V function.

The polydipsia resulting from the POD lesion could be due to the increased sodium retention and chronically increased plasma osmolality. In fact the increased water intake seen in POD rats was clearly associated with an increase in plasma osmolality and the plasma sodium concentration in this study. With respect to these increases in plasma osmolality and plasma sodium concentration, extracellular dehydration due to hypovolemia may not be a cause, as the hematocrit, body weight, and plasma protein levels were comparable in POD and sham-operated rats. Accordingly, it is necessary to consider that the hypernatremia and hyperosmolality following POD lesion may be due to loss of a circulating hormonal factor(s) contributing to the preservation of osmotic equilibrium. Because plasma levels of AVP were not increased in POD rats in spite of hypernatremia and hyperosmolality, and an inappropriate response of AVP secretion to water deprivation for $48 \mathrm{~h}$ was observed, the deficit in fluid and electrolyte regulation following POD appears to be due to a failure to secrete AVP by transection of the supraoptic-neurohypophysial tract. The hypernatremia state seen in POD rats may also be accounted for by a fall in urinary sodium excretion [12]. In this study, these lesioned rats had significantly lower sodium excretion on the first day of surgery and had a delayed recovery in this variable. It may be possible that a neural pathway through the preoptic-hypothalamic region severed by POD lesions mediates central nervous system control of sodium excretion.

The POD rats nearly normalized spontaneous drinking (second and third days of surgery) following a substantial period of polydipsia. From the present studies it is impossible to specify with 
Table 1. Plasma sodium (Na), osmolality (Osm), and arginine vasopres$\sin (\mathrm{AVP})$ values on days 4,7 , and 9 in preoptic-hypothalamic disconnection (POD), medial preoptic-hypothalamic disconnection (mPOD), and sham-operated (Sham) rats

\begin{tabular}{llccc}
\hline & & 4 th & 7 th & $\begin{array}{c}9 \text { th } \\
(\text { after } 48 \mathrm{~h} \mathrm{WR})\end{array}$ \\
\hline Plasma Na & POD & $144 \pm 1 \dagger \dagger$ & $142 \pm 1^{* *+\dagger}$ & $177 \pm 3+\dagger$ \\
$(\mathrm{mmol} / l)$ & mPOD & - & $133 \pm 1$ & - \\
& Sham & $138 \pm 1$ & $134 \pm 1$ & $138 \pm 1$ \\
Plasma Osm & POD & $299 \pm 3 \dagger \dagger$ & $307 \pm 2 * * \dagger$ & $369 \pm 7 \dagger \dagger$ \\
$(\mathrm{mOsm} / \mathrm{kg})$ & mPOD & - & $283 \pm 1$ & - \\
& Sham & $286 \pm 2$ & $286 \pm 1$ & $296 \pm 1$ \\
Plasma AVP & POD & ND & ND & $0.1 \pm 0.1 \dagger \dagger$ \\
$(\mathrm{pmol} / l)$ & mPOD & - & $\mathrm{ND}$ & - \\
& Sham & $0.3 \pm 0.3$ & $\mathrm{ND}$ & $6.3 \pm 1.5$ \\
\hline
\end{tabular}

Values are the means \pm SEM. $* *, P<0.01$ vs. mPOD. $\dagger \dagger, P<0.01$ vs. Sham. WR, water restriction; ND, not detectable.

Table 2. Changes in metabolic variables before and after water restriction (WR) in preoptic-hypothalamic disconnection (POD) rats and sham-operated (Sham) rats

\begin{tabular}{cllcl}
\hline & & $\begin{array}{l}7 \text { th } \\
\text { (before WR) }\end{array}$ & $\begin{array}{c}8 \text { th } \\
\text { (1st day } \\
\text { after WR) }\end{array}$ & $\begin{array}{c}9 \text { th } \\
\text { (2nd day } \\
\text { after WR) }\end{array}$ \\
& & & & \\
\hline Body weight & POD & $264 \pm 8$ & $225 \pm 7^{* *}$ & $213 \pm 6^{* *}$ \\
(g) & Sham & $252 \pm 7$ & $225 \pm 8^{* *}$ & $215 \pm 7^{* *}$ \\
Urine volume & POD & $95.7 \pm 18.2 \dagger \dagger$ & $22.5 \pm 2.2^{* * \dagger \dagger}$ & $6.6 \pm 1.0^{* * \dagger}$ \\
$(\mathrm{ml} / 24 \mathrm{~h})$ & Sham & $19.8 \pm 3.9$ & $9.3 \pm 0.8^{*}$ & $3.5 \pm 0.4^{* *}$ \\
Urine & POD & $367 \pm 65 \dagger \dagger$ & $713 \pm 92^{* *+\dagger}$ & $764 \pm 90^{* * \dagger \dagger}$ \\
osmolality & Sham & $1503 \pm 260$ & $2086 \pm 98^{*}$ & $>3000$ \\
$(\mathrm{mOsm} / \mathrm{kg})$ & & & & \\
Food intake & POD & $19.4 \pm 1.1$ & $5.7 \pm 1.1^{* *+\dagger}$ & $0.9 \pm 0.4^{* *+\dagger}$ \\
$(\mathrm{g} / 24 \mathrm{~h})$ & Sham & $21.1 \pm 0.6$ & $11.5 \pm 0.9^{* *}$ & $5.5 \pm 0.4^{* *}$ \\
\hline
\end{tabular}

Values are the means \pm SEM. $*, * *, P<0.05,0.01$ vs. 7 th day within groups. $\dagger$, $\dagger \dagger, P<0.05,0.01$ vs. Sham on the same day.

certainty the cause of normalization of water intake. However, the activation of other compensatory mechanisms, the influence of surgical stress, or a functional reorganization of remaining neural elements are possible candidates. In addition, POD rats were capable of producing relatively concentrated urine $(764 \pm 90 \mathrm{mOsm} / \mathrm{kg})$ after 48 $\mathrm{h}$ of water restriction compared with value before water restriction $(367 \pm 65 \mathrm{mOsm} / \mathrm{kg})$, although from the results of the present studies we cannot clearly explain what mechanisms can produce this relatively concentrated urine.

Bearler et al. [6] have previously demonstrated that coronal knife cuts posterior to the OVLT alter fluid and sodium regulation. However, they indicated that AVP secretion in lesioned rats was greater than in control rats after water deprivation for $48 \mathrm{~h}$. Since their microknife cuts spared the supraoptic-neurohypophysial pathway, differences from the present studies may be due, at least in part, to a sufficient capacity to secrete AVP in response to osmotic stimulation.

In summary, it is suggested that the efferent fibers running caudally from the AV3V region are not involved in mediation of the hydromineral regulation of the $A V 3 \mathrm{~V}$. 


\section{Acknowledgements}

This work was supported by NHLBI grant HL
6835 and a grant from the Division of Research Resources of NIH for the PROPHET Computer System, and the Reinberger Foundation.

\section{References}

1. Johnson AK, Buggy J (1978) Periventricular preoptic-hypothalamus is vital for thirst and normal water economy. Am J Physiol 234: R122-R129.

2. Brody MJ, Fink GD, Buggy J, Haywood JR, Gordon FJ, Johnson AK (1978) The role of the anteroventral third ventricle $(\mathrm{AV} 3 \mathrm{~V})$ region in experimental hypertension. Circ Res 43 (suppl 1): I-2-I-13.

3. Brody MJ, Johnson AK (1980) Role of the anteroventral third ventricle region in fluid and electrolyte balance, arterial pressure regulation, and hypertension. In: Martini L, Ganong WF (eds) Frontiers in Neuroendocrinology. Raven Press, New York: 249-292.

4. Johnson AK (1985) The periventricular anteroventral third ventricle $(\mathrm{AV} 3 \mathrm{~V})$ : Its relationship with the subfornical organ and neural systems involved in maintaining body fluid homeostasis. Brain Res Bull 15: 595-601.

5. Bealer SL, Carithers J, Johnson AK (1984) Fluid regulation, body weight and drinking responses following hypothalamic knife cuts. Brain Res 305 : 239-245.

6. Bealer SL, Crofton JT, Share L (1983) Hypothalamic knife cuts alter fluid regulation, vasopressin secretion, and natriuresis during water deprivation. Neuroendocrinology 36: 364-370.

7. Castro AL, Almeida EF, Vadenal R, Lopes OU
(1983) Effects of anterior hypothalamic disconnection on the evolution of Goldblatt renal hypertension: a dual response. Hypertension 5 (suppl V): $\mathrm{V}-85-\mathrm{V}-89$.

8. Halasz B, Pupp L (1965) Hormone secretion of the anterior pituitary gland after physical interruption of all nervous pathways to the hypophysiotrophic area. Endocrinology 77: 553-562.

9. Marson O, Chernicky CL, Barnes KL, Diz DI, Slugg RM, Ferrario CM (1985) The anteroventral third ventricle region: participation in the regulation of blood pressure in conscious dogs. Hypertension 7 (suppl I): I-80-I-87.

10. Carithers J, Bealer SL, Brody MJ, Johnson AK (1980) Fine structural evidence of degeneration in supraoptic nucleus and subfornical organ of rats with lesions in the anteroventral third ventricle. Brain Res 201: 1-12.

11. Wilkin LD, Druber KA, Johnson AK (1986) Changes in magnocellular-neurohypophyseal vasopressin following anteroventral third ventricle (AV3V) lesions. J Cardiovasc Pharmacol 8: S-70-S75.

12. Bealer SL, Haywood JR, Gruber KA, Buckalew Jr VM, Fink GD, Brody MJ, Johnson AK (1983) Preoptic-hypothalamic periventricular lesions reduce natriuresis to volume expansion. Am J Physiol 244: R51-R57. 